\title{
Boundedness for commutators of fractional integrals on Herz-Morrey spaces with variable exponent
}

\author{
Jianglong $\mathrm{Wu}$
}

\begin{abstract}
In this paper, some boundedness for commutators of fractional integrals is obtained on Herz-Morrey spaces with variable exponent applying some properties of variable exponent and bounded mean oscillation (BMO) functions.
\end{abstract}

\section{Introduction}

Function spaces with variable exponent are being watched with keen interest not only in real analysis but also in partial differential equations and in applied mathematics because they are applicable to the modeling of electrorheological fluids and image restoration. The theory of function spaces with variable exponent has rapidly made progress in the past 20 years since some elementary properties were established by Kováčik and Rákosník [17]. One of the main problems in the theory is the boundedness of the Hardy-Littlewood maximal operator on variable Lebesgue spaces. By virtue of the fine works [4]-[19], [21], [20], [22], [23], some important conditions on the variable exponent, for example, the log-Hölder conditions, have been obtained.

The class of Herz spaces arose from the study on characterization of multipliers on the classical Hardy spaces. And the homogeneous Herz-Morrey spaces $M \dot{K}_{p, q}^{\alpha, \lambda}\left(\mathbb{R}^{n}\right)$ coordinate with the homogeneous Herz space $\dot{K}_{q}^{\alpha, p}\left(\mathbb{R}^{n}\right)$ when $\lambda=0$. One of the important problems on Herz spaces and Herz-Morrey spaces is the boundedness of sublinear operators. Hernández and Yang [11], Li and Yang [19], and $\mathrm{Lu}$ and Yang [21] have proved that if a sublinear operator $T$ is bounded on $L^{p}\left(\mathbb{R}^{n}\right)$ and satisfies the size condition

$$
|T f(x)| \leq C \int_{\mathbb{R}^{n}} \frac{|f(y)|}{|x-y|^{n}} \mathrm{~d} y
$$

Kyoto Journal of Mathematics, Vol. 54, No. 3 (2014), 483-495

DOI 10.1215/21562261-2693397, (C) 2014 by Kyoto University

Received July 2, 2012. Revised February 18, 2013. Accepted February 28, 2013.

2010 Mathematics Subject Classification: Primary 47B47, 42B20, $42 \mathrm{~B} 35$.

This work was supported in part by the Project for Department of Education of Heilongjiang Province no. 12531720, the Project of Mudanjiang Normal University no. SY201313 and National Science Foundation no. 11161042 of China. 
for all $f \in L^{1}\left(\mathbb{R}^{n}\right)$ with compact support and almost everywhere $x \notin \operatorname{supp} f$, then $T$ is bounded on the homogeneous Herz space $\dot{K}_{q}^{\alpha, p}\left(\mathbb{R}^{n}\right)$. In $2005, \mathrm{Lu}$ and $\mathrm{Xu}$ [20] established the boundedness for some sublinear operators.

The bounded mean oscillation (BMO) space and the BMO norm are defined, respectively, as follows:

$$
\begin{aligned}
\operatorname{BMO}\left(\mathbb{R}^{n}\right) & =\left\{b \in L_{\mathrm{loc}}^{1}\left(\mathbb{R}^{n}\right):\|b\|_{\mathrm{BMO}\left(\mathbb{R}^{n}\right)}<\infty\right\}, \\
\|b\|_{\mathrm{BMO}\left(\mathbb{R}^{n}\right)} & =\sup _{B: \text { ball }} \frac{1}{|B|} \int_{B}\left|b(x)-b_{B}\right| \mathrm{d} x .
\end{aligned}
$$

The fractional integral $I_{\beta}$ is defined by $I_{\beta}(f)(x)=\int_{\mathbb{R}^{n}} \frac{f(y)}{|x-y|^{n-\beta}} \mathrm{d} y$, the commutator for the fractional integral is defined by $\left[b, I_{\beta}\right] f(x)=b(x) I_{\beta}(f)(x)-I_{\beta}(b f)(x)$, and the $m$ th-order commutator for the fractional integral is defined by

$$
I_{\beta, b}^{m}(f)(x)=\int_{\mathbb{R}^{n}} \frac{f(y)(b(x)-b(y))^{m}}{|x-y|^{n-\beta}} \mathrm{d} y,
$$

where $0<\beta<n, b \in \operatorname{BMO}\left(\mathbb{R}^{n}\right), m \in \mathbb{N}$. It is easy to see that, when $m=1$, $I_{\beta, b}^{m}(f)(x)=\left[b, I_{\beta}\right] f(x)$; and when $m=0, I_{\beta, b}^{m}(f)(x)=I_{\beta}(f)(x)$.

Chanillo [3] initially introduced the commutator $\left[b, I_{\beta}\right]$ with $b \in \mathrm{BMO}$ and proved the boundedness on Lebesgue spaces with constant exponent. In 2010, Izuki [14] generalized this result to the case of variable exponent and considered the boundedness on Herz spaces with variable exponent.

In 2010, Izuki [13] proved the boundedness of some sublinear operators on Herz spaces with variable exponent. And recently Izuki [12], [15] also considered the boundedness of some operators on Herz-Morrey spaces with variable exponent.

Motivated by the studies on the Herz spaces and Lebesgue spaces with variable exponent, the main purpose of this paper is to establish some boundedness for commutators of fractional integrals on Herz-Morrey spaces with variable exponent. Our main tools are some properties of variable exponent and BMO function. And we also note that our results are the generalizations of main theorems for Izuki [14], [15] on Herz space and Herz-Morrey spaces with variable exponent.

Throughout this paper, we will denote by $|S|$ the Lebesgue measure and by $\chi_{S}$ the characteristic function for a measurable set $S \subset \mathbb{R}^{n}$. Given a function $f$, we denote the mean value of $f$ on $S$ by $f_{S}:=\frac{1}{|S|} \int_{S} f(x) \mathrm{d} x$. $C$ denotes a constant that is independent of the main parameters involved but whose value may differ from line to line. For any index $1<q(x)<\infty$, we denote by $q^{\prime}(x)$ its conjugate index, namely, $q^{\prime}(x)=\frac{q(x)}{q(x)-1}$. For $A \sim D$, we mean that there is a constant $C>0$ such that $C^{-1} D \leq A \leq C D$.

\section{Preliminaries and lemmas}

In this section, we give the definition of Lebesgue and Herz-Morrey spaces with variable exponent and state their properties. Let $E$ be a measurable set in $\mathbb{R}^{n}$ with $|E|>0$. We first define Lebesgue spaces with variable exponent. 


\section{DEFINITION 2.1}

Let $q(\cdot): E \rightarrow[1, \infty)$ be a measurable function.

(1) The Lebesgue spaces with variable exponent $L^{q(\cdot)}(E)$ are defined by

$$
\begin{aligned}
L^{q(\cdot)}(E)= & \left\{f \text { is measurable function: } \int_{E}\left(\frac{|f(x)|}{\eta}\right)^{q(x)} \mathrm{d} x<\infty\right. \\
& \text { for some constant } \eta>0\} .
\end{aligned}
$$

(2) The space $L_{\text {loc }}^{q(\cdot)}(E)$ is defined by

$$
\begin{aligned}
L_{\mathrm{loc}}^{q(\cdot)}(E)= & \left\{f \text { is measurable function: } f \in L^{q(\cdot)}(K)\right. \\
& \text { for all compact subsets } K \subset E\} .
\end{aligned}
$$

The Lebesgue space $L^{q(\cdot)}(E)$ is a Banach space with the norm defined by

$$
\|f\|_{L^{q(\cdot)}(E)}=\inf \left\{\eta>0: \int_{E}\left(\frac{|f(x)|}{\eta}\right)^{q(x)} \mathrm{d} x \leq 1\right\} .
$$

Now, we define two classes of exponent functions. Given a function $f \in$ $L_{\text {loc }}^{1}(E)$, the Hardy-Littlewood maximal operator $M$ is defined by

$$
M f(x)=\sup _{r>0} r^{-n} \int_{B(x, r) \cap E}|f(y)| \mathrm{d} y \quad(x \in E),
$$

where $B(x, r)=\left\{y \in \mathbb{R}^{n}:|x-y|<r\right\}$.

\section{DEFINITION 2.2}

(1) The set $\mathscr{P}\left(\mathbb{R}^{n}\right)$ consists of all measurable functions $q(\cdot)$ satisfying

$$
1<\underset{x \in \mathbb{R}^{n}}{\operatorname{essinf}} q(x)=q_{-}, \quad q_{+}=\underset{x \in \mathbb{R}^{n}}{\operatorname{essup}} q(x)<\infty .
$$

(2) The set $\mathscr{B}\left(\mathbb{R}^{n}\right)$ consists of all measurable functions $q(\cdot) \in \mathscr{P}\left(\mathbb{R}^{n}\right)$ satisfying that the Hardy-Littlewood maximal operator $M$ is bounded on $L^{q(\cdot)}\left(\mathbb{R}^{n}\right)$.

Next we define the Herz-Morrey spaces with variable exponent. Let $B_{k}=B(0$, $\left.2^{k}\right)=\left\{x \in \mathbb{R}^{n}:|x| \leq 2^{k}\right\}, A_{k}=B_{k} \backslash B_{k-1}$, and $\chi_{k}=\chi_{A_{k}}$ for $k \in \mathbb{Z}$.

\section{DEFINITION 2.3}

Let $\alpha \in \mathbb{R}, 0 \leq \lambda<\infty, 0<p<\infty$, and $q(\cdot) \in \mathscr{P}\left(\mathbb{R}^{n}\right)$. The Herz-Morrey space with variable exponent $M \dot{K}_{p, q(\cdot)}^{\alpha, \lambda}\left(\mathbb{R}^{n}\right)$ is defined by

$$
M \dot{K}_{p, q(\cdot)}^{\alpha, \lambda}\left(\mathbb{R}^{n}\right)=\left\{f \in L_{\mathrm{loc}}^{q(\cdot)}\left(\mathbb{R}^{n} \backslash\{0\}\right):\|f\|_{M \dot{K}_{p, q(\cdot)}^{\alpha, \lambda}\left(\mathbb{R}^{n}\right)}<\infty\right\},
$$

where

$$
\|f\|_{M \dot{K}_{p, q(\cdot)}^{\alpha, \lambda}\left(\mathbb{R}^{n}\right)}=\sup _{k_{0} \in \mathbb{Z}} 2^{-k_{0} \lambda}\left(\sum_{k=-\infty}^{k_{0}} 2^{k \alpha p}\left\|f \chi_{k}\right\|_{L^{q(\cdot)}\left(\mathbb{R}^{n}\right)}^{p}\right)^{1 / p} .
$$


Compare the Herz-Morrey space with variable exponent $M \dot{K}_{p, q(\cdot)}^{\alpha, \lambda}\left(\mathbb{R}^{n}\right)$ with the Herz space with variable exponent $\dot{K}_{q(\cdot)}^{\alpha, p}\left(\mathbb{R}^{n}\right)$ (see [12]), where

$$
\dot{K}_{q(\cdot)}^{\alpha, p}\left(\mathbb{R}^{n}\right)=\left\{f \in L_{\text {loc }}^{q(\cdot)}\left(\mathbb{R}^{n} \backslash\{0\}\right): \sum_{k=-\infty}^{\infty} 2^{k \alpha p}\left\|f \chi_{k}\right\|_{L^{q(\cdot)}\left(\mathbb{R}^{n}\right)}^{p}<\infty\right\} .
$$

Obviously, $M \dot{K}_{p, q(\cdot)}^{\alpha, 0}\left(\mathbb{R}^{n}\right)=\dot{K}_{q(\cdot)}^{\alpha, p}\left(\mathbb{R}^{n}\right)$.

When $\lambda=0$, we can see that our results below generalize the result in the setting of the Herz space with variable exponent, which was proved by Izuki in [14]. So in this paper, we only give the result when $\lambda>0$.

Almeida and Drihem [1] discussed the boundedness of a wide class of sublinear operators, including maximal, potential, and Calderón-Zygmund operators, on variable Herz spaces $K_{q(\cdot)}^{\alpha(\cdot), p}\left(\mathbb{R}^{n}\right)$ and $\dot{K}_{q(\cdot)}^{\alpha(\cdot), p}\left(\mathbb{R}^{n}\right)$. Meanwhile, they also establish Hardy-Littlewood-Sobolev theorems for fractional integrals on variable Herz spaces. In this paper, the author only considers the Herz-Morrey space $M \dot{K}_{p, q(\cdot)}^{\alpha(\cdot), \lambda}\left(\mathbb{R}^{n}\right)$ with variable exponent $q(\cdot)$ but fixed $\alpha \in \mathbb{R}$ and $p \in(0, \infty)$. However, for the case when the exponent $\alpha(\cdot)$ is variable as well, we can refer to further work of the author.

Next we state some properties of variable exponents. Cruz-Uribe, Fiorenza, and Neugebauer [6] and Nekvinda [22] proved the following sufficient conditions independently. Moreover, we note that Diening [7] proved the following proposition in the case when $E$ is bounded, and Nekvinda [22] gave a more general condition in place of (2).

\section{PROPOSITION 2.1}

Suppose that $E$ is an open set. If $q(\cdot) \in \mathscr{P}(E)$ satisfies the inequality

$$
\begin{aligned}
& |q(x)-q(y)| \leq \frac{-C}{\ln (|x-y|)} \quad \text { if }|x-y| \leq 1 / 2, \\
& |q(x)-q(y)| \leq \frac{C}{\ln (e+|x|)} \quad \text { if }|y| \geq|x|
\end{aligned}
$$

where $C>0$ is a constant independent of $x$ and $y$, then we have $q(\cdot) \in \mathscr{B}(E)$.

In order to prove our main theorem, we also need the following result, which is the Hardy-Littlewood-Sobolev theorem on Lebesgue spaces with varible expoonent due to Capone, Cruz-Uribe, and Fiorenza (see [2, Theorem 1.8]). We remark that this result was initially proved by Diening [8] provided that $q_{1}(\cdot)$ is constant outside of a large ball.

PROPOSITION 2.2 (SEE [2])

Suppose that $q_{1}(\cdot) \in \mathscr{P}\left(\mathbb{R}^{n}\right)$ satisfies conditions (1) and (2) in Proposition 2.1. Set $0<\beta<n /\left(q_{1}\right)_{+}$, and define $q_{2}(\cdot)$ by

$$
\frac{1}{q_{1}(x)}-\frac{1}{q_{2}(x)}=\frac{\beta}{n}
$$


Then we have

$$
\left\|I_{\beta} f\right\|_{L^{q_{2}(\cdot)\left(\mathbb{R}^{n}\right)}} \leq C\|f\|_{L^{q_{1}(\cdot)}\left(\mathbb{R}^{n}\right)}
$$

for all $f \in L^{q_{1}(\cdot)}\left(\mathbb{R}^{n}\right)$.

In addition, the following result for the boundedness of $I_{\beta, b}^{m}$ on the Lebesgue spaces with variable exponent will be used in the proof of our main theorem.

\section{PROPOSITION 2.3}

Suppose that $q_{1}(\cdot) \in \mathscr{P}\left(\mathbb{R}^{n}\right)$ satisfies conditions (1) and (2) in Proposition 2.1. Let $m \in \mathbb{N}, 0<\beta<n /\left(q_{1}\right)_{+}$. Define the variable exponent $q_{2}(\cdot)$ by

$$
\frac{1}{q_{1}(x)}-\frac{1}{q_{2}(x)}=\frac{\beta}{n} \text {. }
$$

Then $I_{\beta, b}^{m}$ is bounded from $L^{q_{1}(\cdot)}\left(\mathbb{R}^{n}\right)$ into $L^{q_{2}(\cdot)}\left(\mathbb{R}^{n}\right)$ for all $f \in L^{q_{1}(\cdot)}\left(\mathbb{R}^{n}\right)$ and $b \in \operatorname{BMO}\left(\mathbb{R}^{n}\right)$.

The idea of the proof for Proposition 2.3 comes from [14, Theorem 1]. We omit the details.

The next lemma describes the generalized Hölder's inequality and the duality of $L^{q(\cdot)}(E)$. The proof is found in [17].

\section{LEMMA 2.1 (SEE [17])}

Suppose that $q(\cdot) \in \mathscr{P}(E)$, then the following statements hold.

(1) For all $f \in L^{q(\cdot)}(E)$ and all $g \in L^{q^{\prime}(\cdot)}(E)$, we have

$$
\int_{E}|f(x) g(x)| \mathrm{d} x \leq r_{q}\|f\|_{L^{q(\cdot)}(E)}\|g\|_{L^{q^{\prime}(\cdot)}(E)},
$$

where $r_{q}=1+1 / q_{-}-1 / q_{+}$(generalized Hölder's inequality).

(2) For all $f \in L^{q(\cdot)}(E)$, we have

$$
\|f\|_{L^{q(\cdot)}(E)} \leq \sup \left\{\int_{E}|f(x) g(x)| \mathrm{d} x:\|g\|_{L^{q^{(}(\cdot)}(E)} \leq 1\right\} .
$$

LEMMA 2.2 (SEE [15])

If $q(\cdot) \in \mathscr{B}\left(\mathbb{R}^{n}\right)$, then there exists a positive constant $\delta \in(0,1)$ and $C>0$ such that

$$
\frac{\left\|\chi_{S}\right\|_{L^{q(\cdot)}\left(\mathbb{R}^{n}\right)}}{\left\|\chi_{B}\right\|_{L^{q(\cdot)}\left(\mathbb{R}^{n}\right)}} \leq C\left(\frac{|S|}{|B|}\right)^{\delta}
$$

holds for all balls $B$ in $\mathbb{R}^{n}$ and all measurable subsets $S \subset B$.

LEMMA 2.3 (SEE [15])

If $q(\cdot) \in \mathscr{B}\left(\mathbb{R}^{n}\right)$, then there exists a positive constant $C>0$ such that

$$
C^{-1} \leq \frac{1}{|B|}\left\|\chi_{B}\right\|_{L^{q(\cdot)}\left(\mathbb{R}^{n}\right)}\left\|\chi_{B}\right\|_{L^{q^{\prime}(\cdot)}\left(\mathbb{R}^{n}\right)} \leq C
$$

for all balls $B$ in $\mathbb{R}^{n}$. 
LEMMA 2.4 (SEE [13])

Let $b \in \operatorname{BMO}\left(\mathbb{R}^{n}\right), m \in \mathbb{N}$, and $i, j \in \mathbb{Z}$ with $i<j$. Then we have

$$
\begin{aligned}
C^{-1}\|b\|_{\mathrm{BMO}\left(\mathbb{R}^{n}\right)}^{m} & \leq \sup _{B} \frac{1}{\left\|\chi_{B}\right\|_{L^{q(\cdot)}\left(\mathbb{R}^{n}\right)}}\left\|\left(b-b_{B}\right)^{m} \cdot \chi_{B}\right\|_{L^{q(\cdot)}\left(\mathbb{R}^{n}\right)} \\
& \leq C\|b\|_{\mathrm{BMO}\left(\mathbb{R}^{n}\right)}^{m}, \\
\left\|\left(b-b_{B_{i}}\right)^{m} \cdot \chi_{B_{j}}\right\|_{L^{q(\cdot)}\left(\mathbb{R}^{n}\right)} & \leq C(j-i)^{m}\|b\|_{\mathrm{BMO}\left(\mathbb{R}^{n}\right)}^{m}\left\|\chi_{B_{j}}\right\|_{L^{q(\cdot)}\left(\mathbb{R}^{n}\right)} .
\end{aligned}
$$

The above result is proved by Izuki [13]. We remark that Lemma 2.4 is a generalization of well-known properties for BMO spaces.

\section{Main theorem and its proof}

In this section we prove the boundedness for the higher-order commutator of fractional integrals on Herz-Morrey spaces with variable exponent under some conditions.

Let $q(\cdot) \in \mathscr{P}\left(\mathbb{R}^{n}\right)$ satisfy conditions (1) and (2) in Proposition 2.1. Then so does $q^{\prime}(\cdot)$. In particular, we can see that $q(\cdot), q^{\prime}(\cdot) \in \mathscr{B}\left(\mathbb{R}^{n}\right)$ from Proposition 2.1. Therefore applying Lemma 2.2 when $q_{1}(\cdot), q_{2}(\cdot) \in \mathscr{P}\left(\mathbb{R}^{n}\right)$, we can take a constant $\delta_{1} \in\left(0,1 /\left(q_{2}^{\prime}\right)_{+}\right), \delta_{2} \in\left(0,1 /\left(q_{1}\right)_{+}\right)$such that

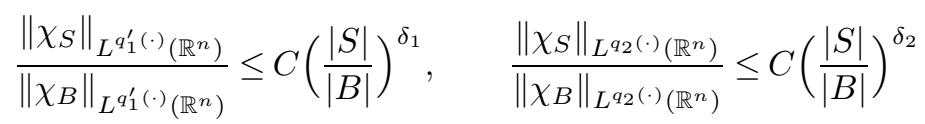

for all balls $B$ in $\mathbb{R}^{n}$ and all measurable subsets $S \subset B$.

Our main result can be stated as follows.

\section{THEOREM 3.1}

Suppose that $q_{1}(\cdot) \in \mathscr{P}\left(\mathbb{R}^{n}\right)$ satisfies conditions (1) and (2) in Proposition 2.1. Define the variable exponent $q_{2}(\cdot)$ by

$$
\frac{1}{q_{1}(x)}-\frac{1}{q_{2}(x)}=\frac{\beta}{n}
$$

Let $m \in \mathbb{N}, 0<p_{1} \leq p_{2}<\infty, \lambda>0,0<\beta<n /\left(q_{1}\right)_{+}, \lambda-n \delta_{2}<\alpha<\lambda+n \delta_{1}$, where $\delta_{1} \in\left(0,1 /\left(q_{1}^{\prime}\right)_{+}\right)$and $\delta_{2} \in\left(0,1 /\left(q_{2}\right)_{+}\right)$are the constants appearing in (3). Then $I_{\beta, b}^{m} \quad$ is bounded from $M \dot{K}_{p_{1}, q_{1}(\cdot)}^{\alpha, \lambda}\left(\mathbb{R}^{n}\right)$ into $M \dot{K}_{p_{2}, q_{2}(\cdot)}^{\alpha, \lambda}\left(\mathbb{R}^{n}\right)$ for all $f \in$ $M \dot{K}_{p_{1}, q_{1}(\cdot)}^{\alpha, \lambda}\left(\mathbb{R}^{n}\right)$ and $b \in \operatorname{BMO}\left(\mathbb{R}^{n}\right)$.

Proof

For all $f \in M \dot{K}_{p_{1}, q_{1}(\cdot)}^{\alpha, \lambda}\left(\mathbb{R}^{n}\right)$ and $\forall b \in \operatorname{BMO}\left(\mathbb{R}^{n}\right)$, if we denote $f_{j}:=f \cdot \chi_{j}=f \cdot \chi_{A_{j}}$ for each $j \in \mathbb{Z}$, then we can write

$$
f(x)=\sum_{j=-\infty}^{\infty} f(x) \cdot \chi_{j}(x)=\sum_{j=-\infty}^{\infty} f_{j}(x) .
$$


Because of $0<p_{1} / p_{2} \leq 1$, we apply the inequality

$$
\left(\sum_{i=-\infty}^{\infty}\left|a_{i}\right|\right)^{p_{1} / p_{2}} \leq \sum_{i=-\infty}^{\infty}\left|a_{i}\right|^{p_{1} / p_{2}}
$$

and obtain

$$
\begin{aligned}
& \left\|I_{\beta, b}^{m}(f)\right\|_{M K_{p_{2}, q_{2}(\cdot)}^{\alpha, \lambda}}^{p_{1}}\left(\mathbb{R}^{n}\right) \\
& =\sup _{k_{0} \in \mathbb{Z}} 2^{-k_{0} \lambda p_{1}}\left(\sum_{k=-\infty}^{k_{0}} 2^{k \alpha p_{2}}\left\|I_{\beta, b}^{m}(f) \cdot \chi_{k}\right\|_{L^{q_{2}(\cdot)}\left(\mathbb{R}^{n}\right)}^{p_{2}}\right)^{p_{1} / p_{2}} \\
& \leq C \sup _{k_{0} \in \mathbb{Z}} 2^{-k_{0} \lambda p_{1}}\left(\sum_{k=-\infty}^{k_{0}} 2^{k \alpha p_{1}}\left\|I_{\beta, b}^{m}(f) \cdot \chi_{k}\right\|_{L^{q_{2}(\cdot)}\left(\mathbb{R}^{n}\right)}^{p_{1}}\right) \\
& \leq C \sup _{k_{0} \in \mathbb{Z}} 2^{-k_{0} \lambda p_{1}}\left(\sum_{k=-\infty}^{k_{0}} 2^{k \alpha p_{1}}\left(\sum_{j=-\infty}^{k-2}\left\|I_{\beta, b}^{m}\left(f_{j}\right) \cdot \chi_{k}\right\|_{L^{q_{2}(\cdot)}\left(\mathbb{R}^{n}\right)}\right)^{p_{1}}\right) \\
& \quad+C \sup _{k_{0} \in \mathbb{Z}} 2^{-k_{0} \lambda p_{1}}\left(\sum_{k=-\infty}^{k_{0}} 2^{k \alpha p_{1}}\left(\sum_{j=k-1}^{k+1}\left\|I_{\beta, b}^{m}\left(f_{j}\right) \cdot \chi_{k}\right\|_{L^{q_{2}(\cdot)}\left(\mathbb{R}^{n}\right)}\right)^{p_{1}}\right) \\
& \quad+C \sup _{k_{0} \in \mathbb{Z}} 2^{-k_{0} \lambda p_{1}}\left(\sum_{k=-\infty}^{k_{0}} 2^{k \alpha p_{1}}\left(\sum_{j=k+2}^{\infty}\left\|I_{\beta, b}^{m}\left(f_{j}\right) \cdot \chi_{k}\right\|_{L^{q_{2}(\cdot)}\left(\mathbb{R}^{n}\right)}\right)^{p_{1}}\right) \\
& =C\left(E_{1}+E_{2}+E_{3}\right) .
\end{aligned}
$$

First we estimate $E_{2}$. Using Proposition 2.3, we have

$$
\begin{aligned}
E_{2} & =\sup _{k_{0} \in \mathbb{Z}} 2^{-k_{0} \lambda p_{1}}\left(\sum_{k=-\infty}^{k_{0}} 2^{k \alpha p_{1}}\left(\sum_{j=k-1}^{k+1}\left\|I_{\beta, b}^{m}\left(f_{j}\right) \cdot \chi_{k}\right\|_{L^{q_{2}(\cdot)}\left(\mathbb{R}^{n}\right)}\right)^{p_{1}}\right) \\
& \leq C\|b\|_{\mathrm{BMO}\left(\mathbb{R}^{n}\right)}^{m p_{1}} \sup _{k_{0} \in \mathbb{Z}} 2^{-k_{0} \lambda p_{1}}\left(\sum_{k=-\infty}^{k_{0}} 2^{k \alpha p_{1}}\left(\sum_{j=k-1}^{k+1}\left\|f_{j} \cdot \chi_{k}\right\|_{L^{q_{1}(\cdot)}\left(\mathbb{R}^{n}\right)}\right)^{p_{1}}\right) \\
& \leq C\|b\|_{\mathrm{BMO}\left(\mathbb{R}^{n}\right)}^{m p_{1}} \sup _{k_{0} \in \mathbb{Z}} 2^{-k_{0} \lambda p_{1}}\left(\sum_{k=-\infty}^{k_{0}} 2^{k \alpha p_{1}}\left\|f_{j} \cdot \chi_{k}\right\|_{L^{q_{1}(\cdot)}\left(\mathbb{R}^{n}\right)}^{p_{1}}\right) \\
& =C\|b\|_{\mathrm{BMO}\left(\mathbb{R}^{n}\right)}^{m p_{1}}\|f\|_{M \dot{K}_{p_{1}, q_{1}(\cdot)}^{\alpha, \lambda}\left(\mathbb{R}^{n}\right)}^{p_{1}}
\end{aligned}
$$

Now we consider $E_{1}$. Note that when $x \in A_{k}, j \leq k-2$, and $y \in A_{j}$, then $|x-y| \backsim|x|, 2|y| \leq|x|$. Therefore, using the generalized Hölder's inequality (see Lemma 2.1(1)), we have

$$
\begin{aligned}
& \left|I_{\beta, b}^{m}\left(f_{j}\right)(x) \cdot \chi_{k}(x)\right| \\
& \quad \leq C \int_{A_{j}} \frac{\left|f_{j}(y)\right||b(x)-b(y)|^{m}}{|x-y|^{n-\beta}} \mathrm{d} y \cdot \chi_{k}(x)
\end{aligned}
$$




$$
\begin{aligned}
\leq & C 2^{k(\beta-n)} \int_{A_{j}}\left|f_{j}(y)\right||b(x)-b(y)|^{m} \mathrm{~d} y \cdot \chi_{k}(x) \\
\leq & C 2^{k(\beta-n)}\left(\left|b(x)-b_{B_{j}}\right|^{m} \int_{A_{j}}\left|f_{j}(y)\right| \mathrm{d} y+\int_{A_{j}}\left|f_{j}(y)\right|\left|b(y)-b_{B_{j}}\right|^{m} \mathrm{~d} y\right) \cdot \chi_{k}(x) \\
\leq & C 2^{k(\beta-n)}\left\|f_{j}\right\|_{L^{q_{1}(\cdot)}}{ }_{\left(\mathbb{R}^{n}\right)}\left(\left|b(x)-b_{B_{j}}\right|^{m}\left\|\chi_{j}\right\|_{L^{q_{1}^{\prime}(\cdot)}{ }_{\left(\mathbb{R}^{n}\right)}}\right. \\
& \left.+\left\|\left(b-b_{B_{j}}\right)^{m} \chi_{j}\right\|_{L^{q_{1}^{(}(\cdot)}\left(\mathbb{R}^{n}\right)}\right) \cdot \chi_{k}(x) .
\end{aligned}
$$

Thus, from Lemma 2.4, and noting that $\left\|\chi_{i}\right\|_{L^{s(\cdot)}\left(\mathbb{R}^{n}\right)} \leq\left\|\chi_{B_{i}}\right\|_{L^{s(\cdot)}\left(\mathbb{R}^{n}\right)}$, it follows that

(4)

$$
\begin{aligned}
& \left\|I_{\beta, b}^{m}\left(f_{j}\right) \cdot \chi_{k}\right\|_{L^{q_{2}(\cdot)}\left(\mathbb{R}^{n}\right)} \\
& \leq C 2^{k(\beta-n)}\left\|f_{j}\right\|_{L^{q_{1}(\cdot)}\left(\mathbb{R}^{n}\right)}\left(\left\|\left(b-b_{B_{j}}\right)^{m} \chi_{k}\right\|_{L^{q_{2}(\cdot)}\left(\mathbb{R}^{n}\right)}\left\|\chi_{j}\right\|_{L^{q_{1}^{\prime}(\cdot)}}\left(\mathbb{R}^{n}\right)\right. \\
& \left.+\left\|\left(b-b_{B_{j}}\right)^{m} \chi_{j}\right\|_{L^{q_{1}^{\prime}(\cdot)}\left(\mathbb{R}^{n}\right)}\left\|\chi_{k}\right\|_{L^{q_{2}(\cdot)}\left(\mathbb{R}^{n}\right)}\right) \\
& \leq C 2^{k(\beta-n)}\left\|f_{j}\right\|_{L^{q_{1}(\cdot)}\left(\mathbb{R}^{n}\right)} \\
& \times\left((k-j)^{m}\|b\|_{\mathrm{BMO}\left(\mathbb{R}^{n}\right)}^{m}\left\|\chi_{B_{k}}\right\|_{L^{q_{2}(\cdot)}\left(\mathbb{R}^{n}\right)}\left\|\chi_{j}\right\|_{L^{q_{1}^{\prime}(\cdot)}}{ }_{\left(\mathbb{R}^{n}\right)}\right.
\end{aligned}
$$

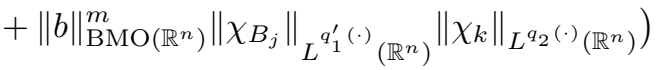

$$
\begin{aligned}
& \leq C 2^{k(\beta-n)}(k-j)^{m}\|b\|_{\mathrm{BMO}\left(\mathbb{R}^{n}\right)}^{m}\left\|f_{j}\right\|_{L^{q_{1}(\cdot)}\left(\mathbb{R}^{n}\right)} \\
& \times\left\|\chi_{B_{j}}\right\|_{L^{q_{1}^{\prime}(\cdot)}}{ }_{\left(\mathbb{R}^{n}\right)}\left\|\chi_{B_{k}}\right\|_{L^{q_{2}(\cdot)}\left(\mathbb{R}^{n}\right)} .
\end{aligned}
$$

Note that $\chi_{B_{k}}(x) \leq C 2^{-k \beta} I_{\beta}\left(\chi_{B_{k}}\right)(x)$ (see [15, p. 350]); by Proposition 2.2 and Lemma 2.3, we obtain

$$
\begin{aligned}
\left\|\chi_{B_{k}}\right\|_{L^{q_{2}(\cdot)}\left(\mathbb{R}^{n}\right)} \leq C 2^{-k \beta}\left\|I_{\beta}\left(\chi_{B_{k}}\right)\right\|_{L^{q_{2}(\cdot)}\left(\mathbb{R}^{n}\right)} \\
\leq C 2^{-k \beta}\left\|\chi_{B_{k}}\right\|_{L^{q_{1}(\cdot)}\left(\mathbb{R}^{n}\right)} .
\end{aligned}
$$

Using Lemma 2.2, Lemma 2.3, (3), and (5), we have

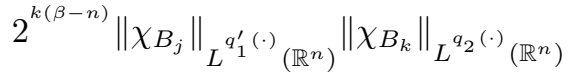

$$
\begin{aligned}
& \leq 2^{k(\beta-n)}\left\|\chi_{B_{j}}\right\|_{L^{q_{1}^{\prime}(\cdot)}} \cdot 2^{-k \beta}\left\|\chi_{B_{k}}\right\|_{L^{q_{1}(\cdot)}\left(\mathbb{R}^{n}\right)} \\
& \leq C\left\|\chi_{B_{j}}\right\|_{L^{q_{1}^{\prime}(\cdot)}{ }_{\left(\mathbb{R}^{n}\right)}} \cdot 2^{-k n}\left\|\chi_{B_{k}}\right\|_{L^{q_{1}(\cdot)}\left(\mathbb{R}^{n}\right)} \\
& \leq C\left\|\chi_{B_{j}}\right\|_{L^{q_{1}^{\prime}(\cdot)}\left(\mathbb{R}^{n}\right)}\left\|\chi_{B_{k}}\right\|_{L^{q_{1}^{\prime}(\cdot)}}^{-1}\left(\mathbb{R}^{n}\right) \\
& =C \frac{\left\|\chi_{B_{j}}\right\|_{L_{1}^{q_{1}^{(\cdot)}}\left(\mathbb{R}^{n}\right)}}{\left\|\chi_{B_{k}}\right\|_{L_{1}^{q_{1}^{\prime}(\cdot)}\left(\mathbb{R}^{n}\right)}} \leq C 2^{(j-k) n \delta_{1}} .
\end{aligned}
$$


On the other hand, note the following fact:

$$
\begin{aligned}
& \left\|f_{j}\right\|_{L^{q_{1}(\cdot)}\left(\mathbb{R}^{n}\right)} \\
& \quad=2^{-j \alpha}\left(2^{j \alpha p_{1}}\left\|f_{j}\right\|_{L^{q_{1}(\cdot)}\left(\mathbb{R}^{n}\right)}^{p_{1}}\right)^{1 / p_{1}} \\
& \quad \leq 2^{-j \alpha}\left(\sum_{i=-\infty}^{j} 2^{i \alpha p_{1}}\left\|f_{i}\right\|_{L^{q_{1}(\cdot)}\left(\mathbb{R}^{n}\right)}^{p_{1}}\right)^{1 / p_{1}} \\
& \quad=2^{j(\lambda-\alpha)}\left(2^{-j \lambda}\left(\sum_{i=-\infty}^{j} 2^{i \alpha p_{1}}\left\|f_{i}\right\|_{L^{q_{1}(\cdot)}\left(\mathbb{R}^{n}\right)}^{p_{1}}\right)^{1 / p_{1}}\right) \\
& \quad \leq C 2^{j(\lambda-\alpha)}\|f\|_{M \dot{K}_{p_{1}, q_{1}(\cdot)}^{\alpha, \lambda}}\left(\mathbb{R}^{n}\right) \cdot
\end{aligned}
$$

Thus, combining (4), (6), and (7), and using $\alpha<\lambda+n \delta_{1}$, it follows that

$$
\begin{aligned}
& E_{1}= \sup _{k_{0} \in \mathbb{Z}} 2^{-k_{0} \lambda p_{1}}\left(\sum_{k=-\infty}^{k_{0}} 2^{k \alpha p_{1}}\left(\sum_{j=-\infty}^{k-2}\left\|I_{\beta, b}^{m}\left(f_{j}\right) \cdot \chi_{k}\right\|_{L^{q_{2}(\cdot)\left(\mathbb{R}^{n}\right)}}\right)^{p_{1}}\right) \\
& \leq C \sup _{k_{0} \in \mathbb{Z}} 2^{-k_{0} \lambda p_{1}} \\
& \times\left(\sum_{k=-\infty}^{k_{0}} 2^{k \alpha p_{1}}\left(\sum_{j=-\infty}^{k-2}(k-j)^{m}\|b\|_{\mathrm{BMO}\left(\mathbb{R}^{n}\right)}^{m}\left\|f_{j}\right\|_{L^{q_{1}(\cdot)}\left(\mathbb{R}^{n}\right)} 2^{-(k-j) n \delta_{1}}\right)^{p_{1}}\right) \\
& \leq C\|b\|_{\mathrm{BMO}\left(\mathbb{R}^{n}\right)}^{m p_{1}}\|f\|_{M \dot{K}_{p_{1}, q_{1}(\cdot)}^{\alpha, \lambda}}^{p_{1}}\left(\mathbb{R}^{n}\right) \\
& \times \operatorname{supp}_{k_{0} \in \mathbb{Z}} 2^{-k_{0} \lambda p_{1}}\left(\sum_{k=-\infty}^{k_{0}} 2^{k \lambda p_{1}}\left(\sum_{j=-\infty}^{k-2}(k-j)^{m} 2^{(k-j)\left(\alpha-\lambda-n \delta_{1}\right)}\right)^{p_{1}}\right) \\
& \leq C\|b\|_{\mathrm{BMO}\left(\mathbb{R}^{n}\right)}^{m p_{1}}\|f\|_{M \dot{K}_{p_{1}, q_{1}(\cdot)}^{\alpha, \lambda}}^{p_{1}}\left(\mathbb{R}^{n}\right) \\
& \sup _{k_{0} \in \mathbb{Z}} 2^{-k_{0} \lambda p_{1}}\left(\sum_{k=-\infty}^{k_{0}} 2^{k \lambda p_{1}}\right) \\
& \leq C\|b\|_{\mathrm{BMO}\left(\mathbb{R}^{n}\right)}^{m p_{1}}\|f\|_{M \dot{K}_{p_{1}, q_{1}(\cdot)}^{\alpha, \lambda}}^{p_{1}}\left(\mathbb{R}^{n}\right)
\end{aligned}
$$

Now, let us turn to the estimate for $E_{3}$. Note that when $x \in A_{k}, j \geq k+2$, and $y \in A_{j}$, then $|x-y| \backsim|y|, 2|x| \leq|y|$. Therefore, using the generalized Hölder's inequality (see Lemma 2.1(1)), we have

$$
\begin{aligned}
& \left|I_{\beta, b}^{m}\left(f_{j}\right)(x) \cdot \chi_{k}(x)\right| \\
& \quad \leq C \int_{A_{j}} \frac{\left|f_{j}(y)\right||b(x)-b(y)|^{m}}{|x-y|^{n-\beta}} \mathrm{d} y \cdot \chi_{k}(x) \\
& \quad \leq C 2^{j(\beta-n)} \int_{A_{j}}\left|f_{j}(y)\right||b(x)-b(y)|^{m} \mathrm{~d} y \cdot \chi_{k}(x) \\
& \quad \leq C 2^{j(\beta-n)}\left(\left|b(x)-b_{B_{k}}\right|^{m} \int_{A_{j}}\left|f_{j}(y)\right| \mathrm{d} y+\int_{A_{j}}\left|f_{j}(y)\right|\left|b(y)-b_{B_{k}}\right|^{m} \mathrm{~d} y\right) \cdot \chi_{k}(x)
\end{aligned}
$$




$$
\begin{aligned}
\leq & C 2^{j(\beta-n)}\left\|f_{j}\right\|_{L^{q_{1}(\cdot)}}{ }_{\left(\mathbb{R}^{n}\right)} \\
& \times\left(\left|b(x)-b_{B_{k}}\right|^{m}\left\|\chi_{j}\right\|_{L^{q_{1}^{\prime}(\cdot)}\left(\mathbb{R}^{n}\right)}+\left\|\left(b-b_{B_{k}}\right)^{m} \chi_{j}\right\|_{L^{q_{1}^{\prime}(\cdot)}{ }_{\left(\mathbb{R}^{n}\right)}}\right) \cdot \chi_{k}(x) .
\end{aligned}
$$

Using Lemma 2.4, it follows that

$$
\begin{aligned}
& \left\|I_{\beta, b}^{m}\left(f_{j}\right) \cdot \chi_{k}\right\|_{L^{q_{2}(\cdot)}\left(\mathbb{R}^{n}\right)} \\
& \leq C 2^{j(\beta-n)}\left\|f_{j}\right\|_{L^{q_{1}(\cdot)}\left(\mathbb{R}^{n}\right)}\left(\left\|\left(b-b_{B_{k}}\right)^{m} \chi_{k}\right\|_{L^{q_{2}(\cdot)}\left(\mathbb{R}^{n}\right)}\left\|\chi_{j}\right\|_{L^{q_{1}^{(\cdot)}}\left(\mathbb{R}^{n}\right)}\right. \\
& \left.+\left\|\left(b-b_{B_{k}}\right)^{m} \chi_{j}\right\|_{L^{q_{1}^{\prime(\cdot)}}}\left\|\chi_{\left.\mathbb{R}^{n}\right)} \chi_{k}\right\|_{L^{q_{2}(\cdot)}\left(\mathbb{R}^{n}\right)}\right) \\
& \text { (8) } \quad \leq C 2^{j(\beta-n)}\left\|f_{j}\right\|_{L^{q_{1}(\cdot)}\left(\mathbb{R}^{n}\right)}\left(\|b\|_{\mathrm{BMO}\left(\mathbb{R}^{n}\right)}^{m}\left\|\chi_{B_{k}}\right\|_{L^{q_{2}(\cdot)}\left(\mathbb{R}^{n}\right)}\left\|\chi_{j}\right\|_{L^{q_{1}^{\prime}(\cdot)}\left(\mathbb{R}^{n}\right)}\right. \\
& \left.+(j-k)^{m}\|b\|_{\mathrm{BMO}\left(\mathbb{R}^{n}\right)}^{m}\left\|\chi_{B_{j}}\right\|_{L^{q_{1}^{(\cdot)}}\left(\mathbb{R}^{n}\right)}\left\|\chi_{k}\right\|_{L^{q_{2}(\cdot)}\left(\mathbb{R}^{n}\right)}\right) \\
& \leq C 2^{j(\beta-n)}(j-k)^{m}\|b\|_{\mathrm{BMO}\left(\mathbb{R}^{n}\right)}^{m}\left\|f_{j}\right\|_{L^{q_{1}(\cdot)}\left(\mathbb{R}^{n}\right)} \\
& \times\left\|\chi_{B_{j}}\right\|_{L^{q_{1}^{\prime}(\cdot)}}\left\|\chi_{\left.\mathbb{R}^{n}\right)}\right\| \chi_{B_{k}} \|_{L^{q_{2}(\cdot)}\left(\mathbb{R}^{n}\right)} .
\end{aligned}
$$

Note that $\chi_{B_{j}}(x) \leq C 2^{-j \beta} I_{\beta}\left(\chi_{B_{j}}\right)(x)$ (see [15, p. 350]), by Proposition 2.2 and Lemma 2.3, we obtain

$$
\begin{aligned}
\left\|\chi_{B_{j}}\right\|_{L^{q_{2}(\cdot)}\left(\mathbb{R}^{n}\right)} \leq C 2^{-j \beta}\left\|I_{\beta}\left(\chi_{B_{j}}\right)\right\|_{L^{q_{2}(\cdot)}\left(\mathbb{R}^{n}\right)} \\
\leq C 2^{-j \beta}\left\|\chi_{B_{j}}\right\|_{L^{q_{1}(\cdot)}\left(\mathbb{R}^{n}\right)} \\
\leq C 2^{-j \beta} 2^{j n}\left\|\chi_{B_{j}}\right\|_{L^{q_{1}^{\prime}(\cdot)}}^{-1}\left(\mathbb{R}^{n}\right)
\end{aligned}
$$

Thus, we have

$$
2^{j(\beta-n)}\left\|\chi_{B_{j}}\right\|_{L^{q_{1}^{\prime}(\cdot)}\left(\mathbb{R}^{n}\right)} \leq C\left\|\chi_{B_{j}}\right\|_{L^{q_{2}(\cdot)}\left(\mathbb{R}^{n}\right)}^{-1} .
$$

Using Lemma 2.2, Lemma 2.3, (3), and (9), we have

$$
\begin{aligned}
2^{j(\beta-n)}\left\|\chi_{B_{j}}\right\|_{L^{q_{1}^{\prime}(\cdot)}\left(\mathbb{R}^{n}\right)}\left\|\chi_{B_{k}}\right\|_{L^{q_{2}(\cdot)}\left(\mathbb{R}^{n}\right)} & \left.\leq C\left\|\chi_{B_{j}}\right\|_{L^{q_{2}(\cdot)}}^{-1} \| \mathbb{R}^{n}\right) \\
& \leq C \frac{\left\|\chi_{B_{k}}\right\|_{L^{q_{2}(\cdot)}} \|_{\left.L^{\mathbb{R}^{n}}\right)}}{\left\|\chi_{B_{j}(\cdot)}\right\|_{L^{q_{2}(\cdot)}\left(\mathbb{R}^{n}\right)}} \\
& \leq C 2^{(k-j) n \delta_{2}} .
\end{aligned}
$$

Thus, combining (7), (8), and (10), and using $\lambda-n \delta_{2}<\alpha$, it follows that

$$
\begin{aligned}
E_{3} & =\sup _{k_{0} \in \mathbb{Z}} 2^{-k_{0} \lambda p_{1}}\left(\sum_{k=-\infty}^{k_{0}} 2^{k \alpha p_{1}}\left(\sum_{j=k+2}^{\infty}\left\|I_{\beta, b}^{m}\left(f_{j}\right) \cdot \chi_{k}\right\|_{L^{q_{2}(\cdot)}\left(\mathbb{R}^{n}\right)}\right)^{p_{1}}\right) \\
& \leq C \sup _{k_{0} \in \mathbb{Z}} 2^{-k_{0} \lambda p_{1}}
\end{aligned}
$$




$$
\begin{aligned}
& \times\left(\sum_{k=-\infty}^{k_{0}} 2^{k \alpha p_{1}}\left(\sum_{j=k+2}^{\infty}(j-k)^{m}\|b\|_{\mathrm{BMO}\left(\mathbb{R}^{n}\right)}^{m}\left\|f_{j}\right\|_{L^{q_{1}(\cdot)}\left(\mathbb{R}^{n}\right)} 2^{-(j-k) n \delta_{2}}\right)^{p_{1}}\right) \\
\leq & C\|b\|_{\mathrm{BMO}\left(\mathbb{R}^{n}\right)}^{m p_{1}}\|f\|_{M \dot{K}_{p_{1}, q_{1}(\cdot)}^{\alpha, \lambda}\left(\mathbb{R}^{n}\right)}^{p_{1}} \\
& \times \sup _{k_{0} \in \mathbb{Z}} 2^{-k_{0} \lambda p_{1}}\left(\sum_{k=-\infty}^{k_{0}} 2^{k \lambda p_{1}}\left(\sum_{j=k+2}^{\infty}(j-k)^{m} 2^{(j-k)\left(\lambda-\alpha-n \delta_{2}\right)}\right)^{p_{1}}\right) \\
\leq & C\|b\|_{\mathrm{BMO}\left(\mathbb{R}^{n}\right)}^{m p_{1}}\|f\|_{M \dot{K}_{p_{1}, q_{1}(\cdot)}^{\alpha, \lambda}\left(\mathbb{R}^{n}\right)}^{p_{k_{0}} \in \mathbb{Z}} 2^{-k_{0} \lambda p_{1}}\left(\sum_{k=-\infty}^{k_{0}} 2^{k \lambda p_{1}}\right) \\
\leq & C\|b\|_{\mathrm{BMO}\left(\mathbb{R}^{n}\right)}^{m p_{1}}\|f\|_{M \dot{K}_{p_{1}, q_{1}(\cdot)}^{\alpha, \lambda}}^{p_{1}}\left(\mathbb{R}^{n}\right)^{\cdot}
\end{aligned}
$$

This finishes the proof of Theorem 3.1.

When $\lambda=0$, our main result also holds on Herz space with variable exponent, and generalizes the result of Izuki [14] (see Theorem 3). When $m=0$, we also improve the result of Izuki [15] (see Theorem 2).

Acknowledgments. The author cordially thanks the referees for their valuable suggestions and useful comments which have led to the improvement of this paper.

\section{References}

[1] A. Almeida and D. Drihem, Maximal, potential and singular type operators on Herz spaces with variable exponents, J. Math. Anal. Appl. 394 (2012), 781-795. MR 2927498. DOI 10.1016/j.jmaa.2012.04.043.

[2] C. Capone, D. Cruz-Uribe, and A. Fiorenza, The fractional maximal operator and fractional integrals on variable $L^{p}$ spaces, Rev. Mat. Iberoam. 23 (2007), 743-770. MR 2414490. DOI 10.4171/RMI/511.

[3] S. Chanillo, A note on commutators, Indiana Univ. Math. J. 31 (1982), 7-16. MR 0642611. DOI 10.1512/iumj.1982.31.31002.

[4] D. Cruz-Uribe, L. Diening, and A. Fiorenza, A new proof of the boundedness of maximal operators on variable Lebesgue spaces, Boll. Unione Mat. Ital. (9) 2 (2009), 151-173. MR 2493649.

[5] D. Cruz-Uribe, A. Fiorenza, J. Martell, and C. Pérez, The boundedness of classical operators on variable $L^{p}$ spaces, Ann. Acad. Sci. Fenn. Math. 31 (2006), 239-264. MR 2210118.

[6] D. Cruz-Uribe, A. Fiorenza, and C. Neugebauer, The maximal function on variable $L^{p}$ spaces, Ann. Acad. Sci. Fenn. Math. 28 (2003), 223-238.

MR 1976842. 
[7] L. Diening, Maximal functions on generalized Lebesgue spaces $L^{p(\cdot)}$, Math. Inequal. Appl. 7 (2004), 245-253. MR 2057643. DOI 10.7153/mia-07-27.

[8] L Riesz potential and Sobolev embeddings on generalized Lebesgue spaces and Sobolev spaces $L^{p(\cdot)}$ and $W^{k, p(\cdot)}$, Math. Nachr. 268 (2004), 31-43. MR 2054530. DOI 10.1002/mana.200310157.

[9] - Maximal functions on Musielak-Orlicz spaces and generalized Lebesgue spaces, Bull. Sci. Math. 129 (2005), 657-700. MR 2166733.

DOI 10.1016/j.bulsci.2003.10.003.

[10] L. Diening, P. Harjulehto, P. Hästö, Y. Mizuta, and T. Shimomura, Maximal functions in variable exponent spaces: Limiting cases of the exponent, Ann. Acad. Sci. Fenn. Math. 34 (2009), 503-522. MR 2553809.

[11] E. Hernández and D. Yang, Interpolation of Herz spaces and applications, Math. Nachr. 205 (1999), 69-87. MR 1709163. DOI 10.1002/mana.3212050104.

[12] M. Izuki, Boundedness of vector-valued sublinear operators on Herz-Morrey spaces with variable exponent, Math. Sci. Res. J. 13 (2009), 243-253. MR 2582455.

[13] B Boundedness of sublinear operators on Herz spaces with variable exponent and application to wavelet characterization, Anal. Math. 36 (2010), 33-50. MR 2606575. DOI 10.1007/s10476-010-0102-8.

[14] Commutators of fractional integrals on Lebesgue and Herz spaces with variable exponent, Rend. Circ. Mat. Palermo (2) 59 (2010), 461-472. MR 2745523. DOI 10.1007/s12215-010-0034-y.

[15] - Fractional integrals on Herz-Morrey spaces with variable exponent, Hiroshima Math. J. 40 (2010), 343-355. MR 2766665.

[16] T. Kopaliani, Infimal convolution and Muckenhoupt $A_{p(\cdot)}$ condition in variable $L^{p}$ spaces, Arch. Math. (Basel) 89 (2007), 185-192. MR 2341730.

DOI 10.1007/s00013-007-2035-4.

[17] O. Kováčik and J. Rákosník, On spaces $L^{p(x)}$ and $W^{k, p(x)}$, Czechoslovak Math. 41 (1991), 592-618. MR 1134951.

[18] A. Lerner, On some questions related to the maximal operator on variable $L^{p}$ spaces, Trans. Amer. Math. Soc. 362 (2010), no. 8, 4229-4242. MR 2608404. DOI 10.1090/S0002-9947-10-05066-X.

[19] X. Li and D. Yang, Boundedness of some sublinear operators on Herz spaces, Illinois J. Math. 40 (1996), 484-501. MR 1407632.

[20] S. Lu and L. Xu, Boundedness of rough singular integral operators on the homogeneous Herz-Morrey spaces, Hokkaido Math. J. 34 (2005), 299-314. MR 2158999.

[21] S. Lu and D. Yang, The decomposition of the weighted Herz spaces and its application, Sci. China Ser. A 38 (1995), 147-158. MR 1338138.

[22] A. Nekvinda, Hardy-Littlewood maximal operator on $L^{p(x)}\left(\mathbb{R}^{n}\right)$, Math. Inequal. Appl. 7 (2004), 255-265. MR 2057644. DOI 10.7153/mia-07-28. 
[23] L. Pick and M. Ružičcka, An example of a space $L^{p(\cdot)}$ on which the Hardy-Littlewood maximal operator is not bounded, Expo. Math. 19 (2001), 369-371. MR 1876258. DOI 10.1016/S0723-0869(01)80023-2.

Department of Mathematics, Mudanjiang Normal University, Mudanjiang 157011, China 